\title{
EFFICIENT FINGERPRINT IMAGE ENHANCEMENT ALGORITHM BASED ON GABOR FILTER
}

\author{
Shaikh Mohammedsayeemuddin ${ }^{1}$, Sima K Gonsai ${ }^{2}$, Dharmesh Vandra ${ }^{3}$ \\ ${ }^{1}$ Student, EC Dept., L.D. College of Engineering, Gujarat Technological University, Ahmedabad, Gujarat, India \\ ${ }^{2}$ Asst. Prof., EC Dept., L.D. College of Engineering, Gujarat Technological University, Ahmedabad, Gujarat, India \\ ${ }^{3}$ Principal, Vedvyas Polytechnic, Himmatnagar, Gujarat, India
}

\begin{abstract}
Fingerprint recognition system is most commonly used biometrics for person identification. The performance of feature extraction algorithm heavily depends on the quality of the fingerprint image. An improved enhancement method based on Gabor filter is presented in this paper. An overlapping block-wise filtering is done in place of conventional pixel wise Gabor filtering. An average frequency of entire image is used instead of each pixel frequency. This reduces the filter complexity and blocking artifacts in an enhanced image. Algorithm is tested on FVC2004 DB1 public fingerprint database. The results show that not only the computation time but accuracy is also improved with our method.
\end{abstract}

Keywords—gabor filter; fingerprint; image enhancement

\section{INTRODUCTION}

Fingerprints are made of ridges and valleys that mostly run parallel [1]. Ideal fingerprint image would have ridges and valley that alternate and flow in locally same direction. Fig. 1(a) shows the example of good quality. But in practice, due to skin conditions (e.g. wet or dry, cut and bruises), noise from sensor, incorrect finger pressure, a significant percentage of fingerprint images are of low quality[2] as show in Fig. 1(b) and (c). Also in many cases, a single fingerprint image contains region of good, medium and poor quality regions as shown in Fig. 2.

Most feature extraction algorithm are based minutiae extraction. Fig. 3 shows the two most common types of minutiae found in fingerprint images. The low quality fingerprint images leads to the following problems in minutiae extraction: (1) a significant number of false minutiae are extracted, (2) a large number of genuine minutiae are
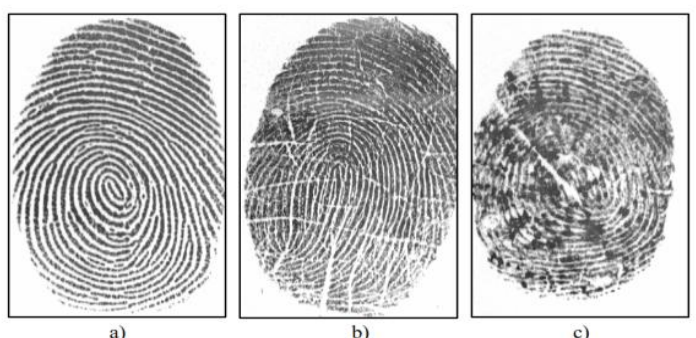

Fig 1 a) A good quality fingerprint; b) a medium quality fingerprints characterized by scratches and ridge breaks; c) a poor quality fingerprint containing a lot of noise.

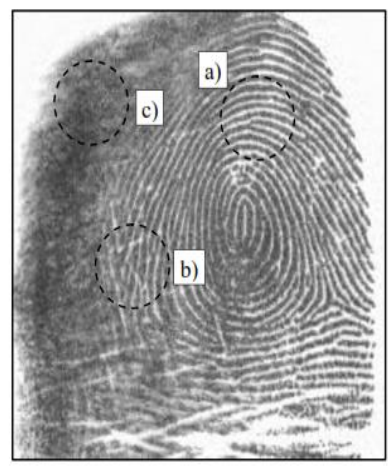

Fig 2 A fingerprint image containing regions of different quality: a) a well-defined region; b) a recoverable region; c) an unrecoverable region.

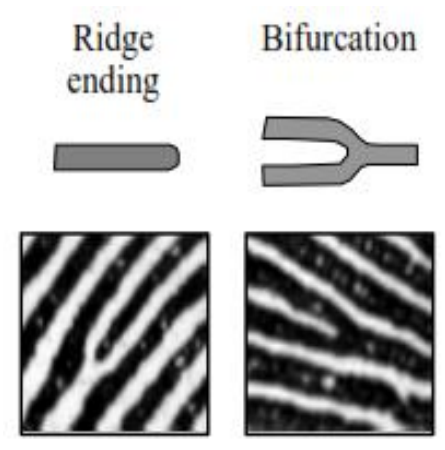

Fig 3 Two most common minutiae types 
missed, and (3) large errors in location of minutiae are introduced. To ensure good performance of feature extraction algorithms in low quality fingerprint images, an enhancement algorithm is necessary for input fingerprint images.

\section{REVIEW}

Many fingerprint image enhancement algorithms are used developed to enhance contrast between ridges and valleys and eliminate noise. There are three basic method for fingerprint image enhancement. (1) Pixel-wise enhancement [3-4], where the new value of each pixel depends only on its previous value and some global parameter (but not on the value of neighboring pixels (2) Contextual filtering [5-8], where filter characteristics change according to local context, In fingerprint image context is most often defined by local ridge frequency and local ridge orientation, and (3) Multi-resolution enhancement [9-14], multi-resolution analysis is used to remove noise from fingerprint images.

\subsection{Traditional Gabor Filter}

Gabor filter is an example of contextual filter, which is widely used in fingerprint applications. Hong et al [3] proposed an effective method for fingerprint image enhancement based on Gabor filters. Gabor filters have both frequency-selective and orientation-selective properties and have optimal joint resolution in both spatial and frequency domains [15-16]. Fig. 4 shows a Gabor filter that is defined by a sinusoidal plane wave (second term of Equation (1)) tapered by a Gaussian (first term in Equation (1)). The even symmetric two dimensional Gabor filter is given by:

$$
g(x, y: \theta, f)=\exp \left\{-\frac{1}{2}\left[\frac{x_{\theta}^{2}}{\sigma_{x}{ }^{2}}+\frac{y_{\theta}{ }^{2}}{\sigma_{y}{ }^{2}}\right]\right\} \cdot \cos \left(2 \pi f \cdot x_{\theta}\right)
$$

Where $\theta$ is the orientation of the filter and

$$
\left(\begin{array}{l}
x_{\theta} \\
y_{\theta}
\end{array}\right)=\left(\begin{array}{cc}
\sin \theta & \cos \theta \\
-\cos \theta & \sin \theta
\end{array}\right)\left(\begin{array}{l}
x \\
y
\end{array}\right)
$$

In Equation (1), $f$ is the frequency of sinusoidal plane wave, and $\sigma_{x}$ and $\sigma_{y}$ are the standard deviations of the Gaussian envelope.

To apply Gabor filter, parameters $\left(\theta, f, \sigma_{x}, \sigma_{y}\right)$ must be specified. The frequency $(f)$ of the filter is determined by the local ridge frequency and the orientation $(\theta)$ is determined by local ridge orientation. For the larger values of $\sigma_{x}, \sigma_{y}$, filter is more robust to noise but they are also likely to create spurious ridges and valleys. And for the smaller values of $\sigma_{x}, \sigma_{y}$, filter is less likely to introduce spurious ridges and valley, but then it will be less effective in removing noise. Hong et al in [4] set $\sigma_{\mathrm{x}}=\sigma_{\mathrm{y}}=4$ based on empirical data.
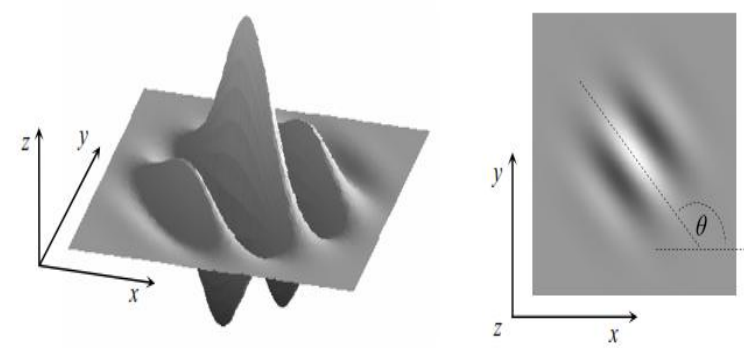

Fig 4 Graphical representation (lateral view and top view) of the Gabor filter defined by the parameters $\theta=135^{\circ}, \mathrm{f}=1 / 5$, and $\sigma \mathrm{x}=\sigma \mathrm{y}=3$.

\subsection{Local Ridge Orientation}

Ridge orientation is generally estimated by computing the gradient of every pixel in an image. The dominant ridge orientation $\theta_{i j}$ in a $17 \times 17$ window $W$ centered at $\left[x_{i}, y_{j}\right]$ is given by following equation[17]:

$$
\begin{gathered}
\theta_{i j}=90^{\circ}+\frac{1}{2} \operatorname{atan} 2\left(2 G_{x y}, G_{x x}-G_{y y}\right), \\
G_{x y}=\sum_{h=-8}^{8} \sum_{k=-8}^{8} \nabla_{x}\left(x_{i}+h, y_{j}+k\right) \cdot \nabla_{y}\left(x_{i}+h, y_{j}+k\right), \\
G_{x x}=\sum_{h=-8}^{8} \sum_{k=-8}^{8} \nabla_{x}\left(x_{i}+h, y_{j}+k\right)^{2}, \\
G_{y y}=\sum_{h=-8}^{8} \sum_{k=-8}^{8} \nabla_{y}\left(x_{i}+h, y_{j}+k\right)^{2}
\end{gathered}
$$

where $\nabla_{x}$ and $\nabla_{y}$ are $x$ - and $y$ - gradient components computed through $3 \times 3$ Sobel operator, and atan 2 calculate the arctangent of the variables $y$ and $x$.

\subsection{Local Ridge Frequency}

Analogous to ridge orientation, ridge frequency can also be computed for each pixel in a fingerprint image. Hong et al [3] estimate local ridge frequency by counting the average number pixels between two consecutive peaks of gray levels along the direction that is normal to the ridge orientation. 

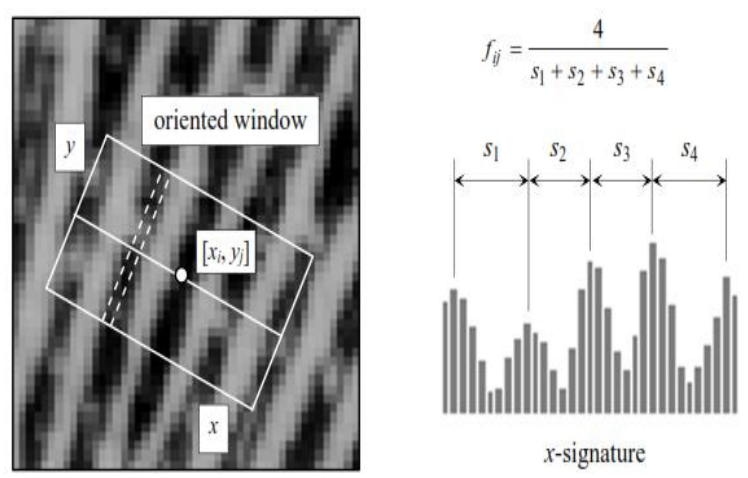

Fig 5 An oriented window centered at $\left[\mathrm{x}_{\mathrm{i}}, \mathrm{y}_{\mathrm{j}}\right]$; the dashed lines show the pixels whose gray-levels are accumulated for a given column of the $\mathrm{x}$-signature. The $\mathrm{x}$-signature on the right clearly exhibits five peaks; the four distances between consecutive peaks are averaged to determine the local ridge frequency.

\subsection{Traditional Gabor Filter Algorithm}

From above discussion traditional Gabor filter algorithm [8] can be summarized as follows:

i. Image normalization to increase the dynamic range.

ii. Compute local ridge orientation.

iii. Compute local ridge frequency.

iv. Gabor filtering using equation (1) based on local ridge orientation and frequency.

So to directly compute the filter output from (1) for any image size, say $100 \times 100$, algorithm will require 10000 iterations, and each of these iterations will require another $625(25 \times 25)$ iterations for convolution operation (assuming $\sigma_{x}=\sigma_{y}=4$ and filter width of 6 times the standard deviation (i.e. 24)) which is computationally extensive task.

\section{PROPOSED ALGORITHM}

Instead of computing filter output for each pixel, in our method filter output for image block of $32 \times 32$. Each next block is overlapped with previous block $16 \times 16$. Local ridge orientation and local ridge frequency are computed as mentioned in previous section. For parameter $f$, average frequency of entire fingerprint $\left(f_{\text {avg }}\right)$ image is used for (1), instead of average frequency of each $32 \times 32$ block; and for parameter, $\theta$, median of orientation of $32 \times 32$ block is used in (1). Using a single frequency value, $f_{\text {avg }}$, for entire image reduces the blocking artifacts in the output image. This makes filter output completely determined by local ridge orientation. $\sigma_{x}=\sigma_{y}=4$ is used, and size of Gabor filter is $25 \times 25$. To overcome the deficiency of block-wise processing some preand post- processing is required for better results. Fig. 6 shows the flowchart of proposed algorithm.

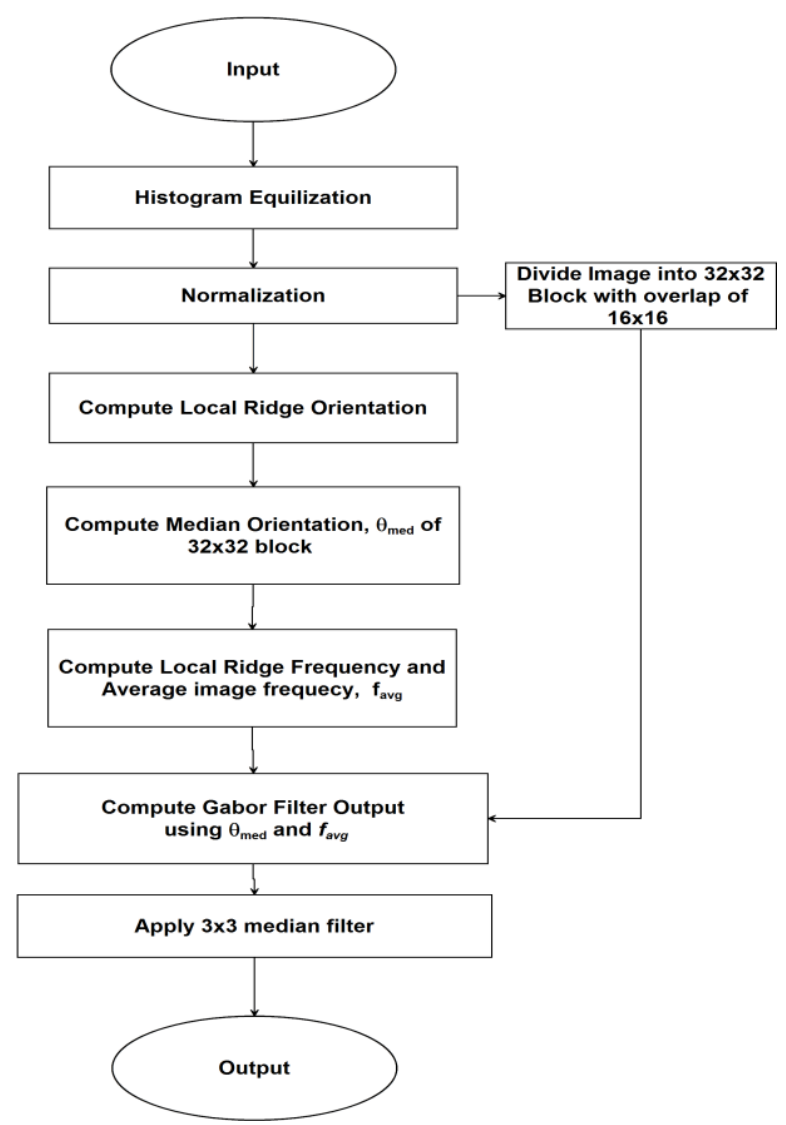

Fig 6 Flowchart of proposed algorithm

\section{ALGORITHM RESULTS}

Proposed algorithm is tested on FVC2004 DB1 public database. Images are of $500 \mathrm{dpi}$ and have size of $640 \times 480$ pixels. It contains images of 10 fingerprints with 8 impression per finger, i.e. total 80 images.

Fig. 7 shows the results of our proposed algorithm along with traditional algorithm after segmentation and binarization. Table I shows the results of performance evaluation for EER (equal error rate) in fingerprint recognition system and average time for image enhancement. Proposed algorithm and traditional Gabor algorithm is applied to same feature extraction algorithm and matching algorithm one by one. Results are compared in Table I.

Table 1: Results

\begin{tabular}{|l|l|c|}
\hline \multirow{2}{*}{\multicolumn{1}{|c|}{ Method }} & \multicolumn{2}{|c|}{ Performance Evaluation } \\
\cline { 2 - 3 } & $\boldsymbol{E E R}$ & $\begin{array}{c}\text { Time cost per } \\
\text { image }\end{array}$ \\
\hline Traditional Gabor & 0.180 & $240 \mathrm{sec}$ \\
\hline Proposed & 0.202 & $7.1 \mathrm{sec}$ \\
\hline
\end{tabular}




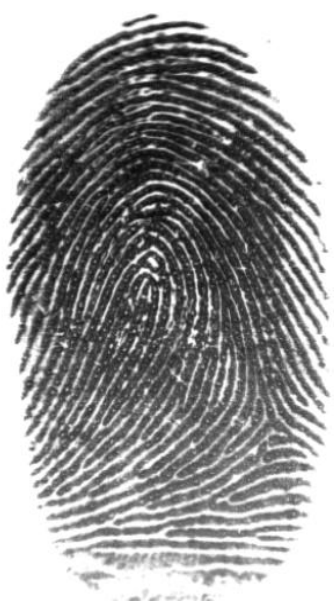

(a)

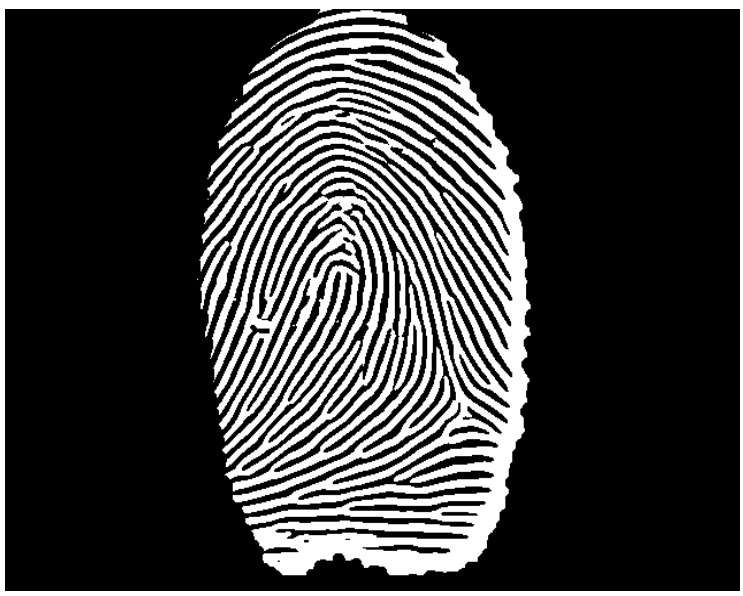

(b)

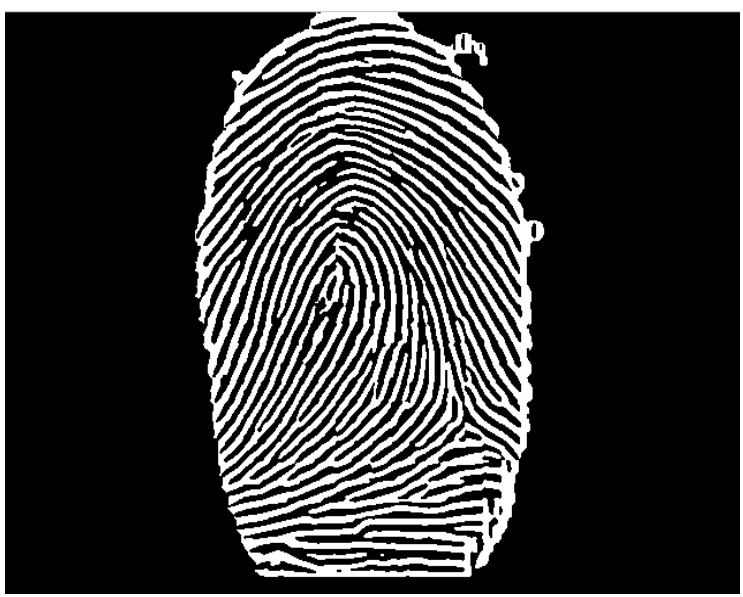

(c)

Fig 7 Results. a) Original image for FVC2004 DB1, b) Result of traditional Gabor filter and c) Result of proposed method.

\section{CONCLUSIONS AND FUTURE WORK}

From Fig. 7 we observe that proposed method give visually comparable results to traditional method. Also EER calculated for FVC2004 DB1 though higher, is close to traditional Gabor filter results. These results are obtained with very high gain in time cost of algorithm. Many improved enhancement algorithm are available in the literature based on Gabor filter [18-26] that uses other techniques to compute filter output. Future work can include comparative analysis with these methods and further improvement can be tried for by postprocessing proposed filter output to eliminate spurious minutiae that are introduced due to block-wise operation.

\section{REFERENCES}

[1] Ashbaugh D.R., Quantitative-Qualitative Friction Ridge Analysis: An Introduction to Basic and Advanced Ridgeology, CRC Press, Boca Raton, FL, 1999.

[2] D. Maltoni, D. Maio, A. K. Jain, and S. Prabhakar,"Handbook of Fingerprint Recognition 2nd Edition", Springer- Verlag, 2009.

[3] Hong L., Wan Y. and Jain A.K., "Fingerprint image enhancement: Algorithms and performance evaluation," IEEE Transactions on Pattern Analysis Machine Intelligence, vol. 20, no. 8, pp. 777-789, 1998.

[4] Greenberg S., Aladjem M., Kogan D. and Dimitrov I., "Fingerprint Image Enhancement Using Filtering Techniques," in Proc. Int. Conf. on Pattern Recognition (15th), vol. 3, pp. 326-329, 2000.

[5] O'Gorman L. and Nickerson J., "Matched Filter Design for Fingerprint Image Enhancement," in Proc. Int. Conf. on Acoustic Speech and Signal Processing, pp. 916-919, 1988..

[6] O'Gorman L. and Nickerson J.V., "An approach to fingerprint filter design," Pattern Recognition, vol. 22, no. 1, pp. 29-38, 1989.

[7] Sherlock B.G., Monro D.M. and Millard K., "Algorithm for enhancing fingerprint images," Electronics Letters, vol. 28, no. 18, pp. 1720, 1992.

[8] Sherlock B.G., Monro D.M. and Millard K., "Fingerprint enhancement by directional Fourier filtering," IEE Proceedings Vision Image and Signal Processing, vol. 141, no. 2, pp. 87-94, 1994.

[9] Almansa A. and Cohen L., "Fingerprint Image Matching by Minimization of a Thin-Plate Energy Using a Two-Step Iterative Algorithm with Auxiliary Variables," in Proc. Workshop on Applications of Computer Vision, pp. 35-40, 2000.

[10] Hsieh C.T., Lai E. and Wang Y.C., "An effective algorithm for fingerprint image enhancement based on wavelet transform," Pattern Recognition, vol. 36, no. 2, pp. 303-312, 2003. 
[11] Cheng J. and Tian J., "Fingerprint enhancement with dyadic scale-space,"Pattern Recognition Letters, vol. 25, no. 11, pp. 1273-1284, 2004.

[12] Fronthaler H., Kollreider K. and Bigun J., "PyramidBased Image Enhancement of Fingerprints," in Proc. Workshop on Automatic Identification Advanced Technologies, pp. 45-50, 2007.

[13] Fronthaler H., Kollreider K. and Bigun J., "Local features for enhancement and minutiae extraction in fingerprints," IEEE Transactions on Image Processing, vol. 17, no. 3, pp. 354-363, 2008.

[14] Mallat S.G., "A theory for multiresolution signal decomposition: The wavelet representation," IEEE Transactions on Pattern Analysis Machine Intelligence, vol. 11, no. 7, pp. 674-693, 1989.

[15] Daugman J.G., "Uncertainty relation for resolution in space, spatial-frequency, and orientation optimized by two-dimensional visual cortical filters," Journal Optical Society American, vol. 2, pp. 1160-1169, 1985.

[16] Jain A.K. and Farrokhnia F., "Unsupervised texture segmentation using Gabor filters," Pattern Recognition, vol. 24, no. 12, pp. 1167-1186, 1991.

[17] Ratha N.K., Chen S.Y. and Jain A.K., "Adaptive flow orientation-based feature extraction in fingerprint images," Pattern Recognition, vol. 28, no. 11, pp. 1657-1672, 1995.

[18] Taoshen Li, Jianhuan Chen, "A fingerprint image enhancement method based on Gabor filter," 2011 Second International Conference on Mechanic Automation and Control Engineering (MACE), pp. $3773-3776,2011$

[19] Saatci, E., Tavsanoglu, V., "Fingerprint image enhancement using CNN Gabor-Type filters, "Proceedings of the 2002 7th IEEE International Workshop on Cellular Neural Networks and Their Applications, 2002. (CNNA 2002)." pp. 377 - 382, 2002.

[20] Hadhoud, M.M., ElKilani, W.S. ; Samaan, M.I., " An adaptive algorithm for fingerprints image enhancement using gabor filters," International Conference on Computer Engineering \& Systems, 2007. ICCES '07., pp. 227 - 236, 2007.

[21] Jingjing Wang, Xiaoyan Sun, "Fingerprint image enhancement using a fast Gabor filter," 2010 8th World Congress on Intelligent Control and Automation (WCICA), ", pp. 6347 - 6350, 2010.

[22] Yi Hu, Xiaojun Jing ; Bo Zhang ; Xifu Zhu, "Low quality fingerprint image enhancement based on Gabor filter," 2010 2nd International Conference on Advanced Computer Control (ICACC), pp. 195 - 199, 2010.

[23] Ching-Han Chen, Kuo-En Chiu, "1-D Gabor Directional Filtering for Low-Quality Fingerprint Image Enhancement," 2006 - 32nd Annual Conference on IEEE Industrial Electronics, IECON, pp. 3466 3470, 2006.
[24] Xian Zhang, Xu-cheng Gong ; Zhong-xing Sun ; Limin Sun, "A novel algorithm for fingerprint orientation extraction and image enhancement based on Gabor filters," 2010 3rd International Congress on Image and Signal Processing (CISP), pp. 1759 - 1762, 2010.

[25] Karimimehr, N., Shirazi, A.A.B. ; Keshavars Bahaghighat, M., "Fingerprint image enhancement using Gabor wavelet transform," 2010 18th Iranian Conference on Electrical Engineering (ICEE), pp. 316 320, 2010.

[26] Keming Mao, Zhiliang Zhu ; Huiyan Jiang, "A Fast Fingerprint Image Enhancement Method," 2010 Third International Joint Conference on Computational Science and Optimization (CSO), pp. 222 - 226, 2010. 